Published in final edited form as:

Proc SPIE Int Soc Opt Eng. 2018 ; 10497: . doi:10.1117/12.2290120.

\title{
Applications and assessment of an excitation-scanning hyperspectral imaging system
}

\author{
Sam A. Mayes ${ }^{1,4}$, Kaysie Moore ${ }^{1}$, Craig Browning ${ }^{1,4}$, Phiwat Klomkaew ${ }^{1}$, Thomas C. \\ Rich $^{2,3}$, Silas J. Leavesley ${ }^{1,2,3}$ \\ ${ }^{1}$ Department of Chemical and Biomolecular Engineering, University of South Alabama \\ ${ }^{2}$ Department of Pharmacology, University of South Alabama \\ ${ }^{3}$ Center for Lung Biology, University of South Alabama \\ ${ }^{4}$ Department of Systems Engineering
}

\section{Abstract}

The majority of microscopic and endoscopic technologies utilize white light illumination. For a number of applications, hyper-spectral imaging can be shown to have significant improvements over standard white-light imaging techniques. This is true for both microscopy and in vivo imaging. However, hyperspectral imaging methods have suffered from slow application times. Often, minutes are required to gather a full imaging stack. Here we will describe and evaluate a novel excitation-scanning hyperspectral imaging system and discuss some applications. We have developed and are optimizing a novel approach called excitation-scanning hyperspectral imaging that provides an order of magnitude increased signal strength. This excitation scanning technique has enabled us to produce a microscopy system capable of high speed hyperspectral imaging with the potential for live video acquisition.

The excitation-scanning hyperspectral imaging technology we developed may impact a range of applications. The current design uses digital strobing to illuminate at 16 wavelengths with millisecond image acquisition time. Analog intensity control enables a fully customizable excitation profile. A significant advantage of excitation-scanning hyperspectral imaging is can identify multiple targets simultaneously in real time. Finally, we are exploring utilizing this technology for a variety of applications ranging from measuring cAMP distribution in three dimensions within a cell to electrophysiology.

\section{Keywords}

Spectroscopy; excitation; hyperspectral; design; tissue imaging; microscopy; endoscopy; LED

\subsection{INTRODUCTION}

Successful cancer treatment and rehabilitation requires early detection. ${ }^{1}$ White-light endoscopy techniques miss up to $27 \%$ of small, flat adenomas. ${ }^{2}$ Hyperspectral imaging acquires spectral information for each point in the image and has been indicated as a potential improvement technology for ex vivo and in vivo microscopy and endoscopy, including lesion detection. ${ }^{3-8}$ Hyperspectral imaging has been shown to provide increased 
sensitivity over white-light imaging for cancer detection. ${ }^{9,10}$ The goal of this work was to develop and deploy a prototype hyperspectral imaging system for microscopy and evaluate and test the system as applied to multi-label detection, high speed imaging, electrophysiology, and five-dimensional (spectral, temporal, $\mathrm{x}, \mathrm{y}$, and $\mathrm{z}$ ) imaging of signaling pathways. In order to increase the speed of our hyperspectral imaging system, we have developed an excitation-scanning technology that allows the potential for real-time hyperspectral imaging. ${ }^{6}$ The hyperspectral illumination system can provide up to 16 wavelengths of light and can be cycled at speeds exceeding $1 \mathrm{~ms}$. These results indicate a potential for hyperspectral image acquisition at over 20 frame per second (e.g., 320 bands imaged per second).

\subsection{METHODS}

\subsection{Excitation-Scanning Hyperspectral Microscope Setup}

Excitation-scanning hyperspectral imaging overcomes some limitations of traditional fluorescence emission-scanning hyperspectral imaging approaches. Signal-to-noise (SNR) is significantly increased by filtering the excitation light instead of the emission. ${ }^{6,11}$ This allows us to collect all emitted fluorescence at each wavelength. The system is comprised of off-the-shelf and in-house fabricated components. The setup of the excitation-scanning hyperspectral microcopy system is similar to a previous version. ${ }^{12}$ The detector used is a sCMOS scientific camera (Zyla 5.5, Andor). This camera was chosen due being capable of 40 frames per second over USB 3.0. The camera was connected to an inverted fluorescence microscope (Nikon Eclipse Ti), which was equipped with custom long-pass dichroic beamsplitters (Semrock). A spectral light source was connected to the illumination input of the microscope via liquid light guide. A custom illumination device was designed and built in-house and delivers up to 16 wavelengths of light into a single liquid light guide. Our current spectral excitation range is from $365 \mathrm{~nm}$ to $525 \mathrm{~nm}$ with roughly $20 \mathrm{~nm}$ bands. Rapid imaging is accomplished using triggered acquisition of the camera and custom control electronics within the light source that deliver digital and analog inputs to each illumination channel. Digital triggering was implemented via TTL signal and National Instruments control cards linked with the camera shutter's firing signal, which allows us to switch the wavelength synchronously with the camera's capture rate. Nikon Instruments (NIS) Elements software was used to control all aspects of the system and conduct preliminary imaging.

\subsection{METHODS AND APPLICATIONS}

\subsection{General Application of Technology}

Excitation-scanning hyperspectral imaging may be an effective technology for identifying multiple fluorescent molecules at high speeds. Our long-term goals are to develop a system that can be used for both microscopic and endoscopic applications. We evaluated the ability of the system to acquire biological fluorescence data by coupling the high-speed illuminator to a microscope via a liquid light guide. In this optical configuration, excitation light from the high-speed hyperspectral illuminator is reflected off a long-pass dichroic beam splitter to the sample through a 60X oil immersion objective. The emitted fluorescence is collected by 
the same 60X objective. This then passes through the long-pass dichroic beamsplitter and is refined using a long-pass emission filter. Finally, an Andor Zyla 5.5 sCMOS camera collects the signal. The camera integrates all the fluorescence as a function of each excitation wavelength and allows the acquisition of excitation-scanning spectral image data. The system is optimized to have a range of potential imaging modalities. We are able collect $1280 \times 1080$ resolution images at exposure ranging from $70 \mathrm{~ms}$ to 1 second depending on the sample. At higher speeds, an image resolution of $976 \times 976$ is achieved with exposure times of 10-50 ms. These modalities for imaging translate directly to video-rate collection speeds. At these resolution, we achieved speeds between $3.5-11$ spectral frames per second. We have previously shown that automated subcellular analysis of second messenger signals for multilabel FRET studies can be performed using hyperspectral microscopic. ${ }^{13-15}$ We expect that this excitation-scanning hyperspectral imaging implementation will allow these same cell signaling studies with greatly increased temporal sampling.

Cell lines used were pulmonary microvascular endothelial cells (PMVECs), human aortic smooth muscle cells (HASMC), Human embryonic kidney cells (HEK293), and human melanoma cells. A variety of labels were evaluated, including: H188 FRET probe containing Turquoise donor and Venus acceptor fluorescent proteins ${ }^{16}$, hydroxyphenyl fluorescein with adenylyl cyclase (HFP-AC10), DRAQ5 (Nuclear Label), NucBlue (Nuclear Label), wheat germ agglutinin (Membrane label), Calcein Green (Cytoplasmic Label), and Tubulin Tracker (Cytoskeleton label). The relative concentrations of each label were also estimated varying single concentrations of label in single and dual label studies to judge relative intensities. All imaging was done using a 60x oil immersion objective to collect fluorescence emission. Each image stack was collected from $365 \mathrm{~nm}$ to $525 \mathrm{~nm}$. Exposure times varied between 10 ms to 1 second per wavelength band depending on the sample.

\subsection{Electrophysiology \& Second Messenger Signaling}

Our novel excitation-scanning hyperspectral imaging system can collect spatial, temporal, and spectral data. This multi-modal data collection will allow optical electrophysiology studies to be conducted that analyze localization, spectral, and temporal information on top of standard patch-clamp voltage readings. This capability to image localized signals using spectral information while simultaneously collecting electrophysiology readings is currently not possible at high temporal resolution with standard electrophysiology equipment. This could facilitate studies of ion channel proteins, neurons, signal diffusion, and a whole host of other important biological processes.

We are currently implementing this system so as to also allow for the collection of data in three dimensions using a piezo stage and our high-speed excitation scanning hyperspectral imaging approach. With a system that can collect image stacks at 11 frames per second, it should be possible to collect data with sufficient multi-dimensional resolution to see cellular diffusion through a cell.

\subsection{RESULTS AND CONCLUSIONS}

The system was tested and calibrated using an integration sphere (FIOS, Ocean Optics) calibrated to a National Institute of Standards and Technology (NIST)-traceable light source 
(LS-1-CAL-INT, Ocean Optics). The integrating sphere was fiber-coupled to a spectrometer (QE6500, Ocean Optics). Spectroradiometric measurements were made before the light path, at each illumination point; and after the light path, at the microscope stage. Using the measured intensities as an optimization parameter, we evaluated and adjusted our relative wavelength power delivery. Wavelength switches was synchronized with camera acquisition through NIS elements software and TTL triggering. Operational speeds were tested, and we collected video and images at the following resolutions: 596×596, 976×976, and $1280 \times 1080$. Video frame rates ranged from 3.5 to 11 frames per second for the samples herein.

NIS Elements was used to identify regions of interest (ROI) corresponding to individual spectral signatures. An average spectrum was extracted from each ROI and used to unmix the images. Image acquisition times varied greatly early on, as we were testing and characterizing different labels and cell types. The images shown below were acquired with exposure times between $10 \mathrm{~ms}$ and $1 \mathrm{~s}$ per spectral band. In this process we were able to identify a variety of labels in a variety of cells with up to 4 simultane ous labels identified in a single acquisition. We believe that with carefu 1 wavelength and label selectio $\mathrm{n}$, multilabel studies should be achievable with high speed acquisition.

Additionally, we acquired spectral video data at $1280 \times 1080$ pixel field s-of-view at speeds up to $\sim 11$ frames per second with 9 channels per frame. This video rat e requires $10 \mathrm{~ms}$ exposure tim e per wavelength channel. B y coupling our real-time, high speed, high quality imaging and video with excitation-scanning hyperspe ctral imaging; we have show $\mathrm{n}$ it is possible to provide multiplex fluorophore detection at video-rate image acquisition. It is our hope to translate these improvements into increase $\mathrm{d}$ specificity and sensitivity in clinical imaging devices. Some example image are seen below in Figure 2 and Figure 3.

\subsection{FUTURE WORK}

Future work will focus on optimizing the light path further to increase our illumination delivery. Additionally, we are identifying more powerful diodes and further software development. Many of the diodes available do not overlap our operational ranges. Additional optical equipment may be needed to collect more of the power output from each diode. We are also developing a fluorescence excitation library of commonly used fluorescent labels. This will be necessary to use the image data for diagnosis and interpretation. Finally, we are looking to develop analysis tools based on collected spectral information to automate detection of regions of interest.

\section{ACKNOWLEDGEMENTS}

The authors give thanks to the Alabama Space Grant Consortium, NSF 1725937, NIH grants P01 HL066299, UL1 TR001417, and the Abraham Mitchell Cancer Research Fund for their support. Support for this project was also provided via a start-up company established by Drs. Leavesley and Rich to commercialize spectral imaging technologies, SpectraCyte, LLC. 


\section{REFERENCES}

[1]. Kester RT, Bedard N, Gao L, and Tkaczyk TS, "Real-time snapshot hyperspectral imaging endoscope,” Journal of Biomedical Optics 16(5), 056005-056005 (2011). [PubMed: 21639573]

[2]. Dekker E, and Fockens P, “Advances in colonic imaging: new endoscopic imaging methods," Journal of Gastroenterology 17(8), 803-808 (2005).

[3]. Vo-Dinh T, “A hyperspectral imaging system for in vivo optical diagnostics," IEEE Engineering in Medicine and Biology Magazine 23(5), 40-49 (2004). [PubMed: 15565798]

[4]. Leavesley S, Jiang Y, Patsekin V, Rajwa B, and Robinson JP, “An excitation wavelength scanning spectral imaging system for preclinical imaging," Review of Scientific Instruments 79(2), 023707-023707-10 (2008). [PubMed: 18315305]

[5]. Leavesley SJ, Annamdevula N, Boni J, Stocker S, Grant K, Troyanovsky B, Rich TC, and Alvarez DF, "Hyperspectral imaging microscopy for identification and quantitative analysis of fluorescently-labeled cells in highly autofluorescent tissue," Journal of Biophotonics 5(1), 67-84 (2012). [PubMed: 21987373]

[6]. Favreau PF, Hernandez C, Heaster T, Alvarez DF, Rich TC, Prabhat P, and Leavesley SJ, "Excitation-scanning hyperspectral imaging microscope," Journal of Biomedical Optics 19(4), 046010-046010 (2014). [PubMed: 24727909]

[7]. Favreau P, Hernandez C, Lindsey AS, Alvarez DF, Rich T, Prabhat P, and Leavesley SJ, “Thin-film tunable filters for hyperspectral fluorescence microscopy," Journal of Biomedical Optics 19(1), 011017-011017 (2013).

[8]. Schultz RA, Nielsen T, Zavaleta JR, Ruch R, Wyatt R, and Garner HR, "Hyperspectral imaging: A novel approach for microscopic analysis," Cytometry 43(4), 239-247 (2001). [PubMed: 11260591]

[9]. Hirsch FR, Prindiville SA, Miller YE, Franklin WA, Dempsey EC, Murphy JR, Bunn PA, and Kennedy TC, "Fluorescence Versus White-Light Bronchoscopy for Detection of Preneoplastic Lesions: a Randomized Study," Journal of the National Cancer Institute 93(18), 1385-1391 (2001). [PubMed: 11562389]

[10]. Haringsma J, Tytgat GNJ, Yano H, Iishi H, Tatsuta M, Ogihara T, Watanabe H, Sato N, Marcon $\mathrm{N}$, et al., "Autofluorescence endoscopy: Feasibility of detection of GI neoplasms unapparent to white light endoscopy with an evolving technology," Gastrointestinal Endoscopy 53(6), 642-650 (2001). [PubMed: 11323596]

[11]. Annamdevula NS, Sweat B, Favreau P, Lindsey AS, Alvarez DF, Rich TC, and Leavesley SJ, "An Approach for Characterizing and Comparing Hyperspectral Microscopy Systems," Sensors 13(7), 9267-9293 (2013). [PubMed: 23877125]

[12]. Mayes SA, Leavesley SJ, and Rich TC, "Excitation-scanning hyperspectral imaging system for microscopic and endoscopic applications," 2016, 97110Z-97110Z-5.

[13]. Leavesley SJ, Britain AL, Cichon LK, Nikolaev VO, and Rich TC, “Assessing FRET using spectral techniques," Cytometry. Part A: The Journal of the International Society for Analytical Cytology 83(10), 898-912 (2013).

[14]. Leavesley SJ, Nakhmani A, Gao Y, and Rich TC, “Automated image analysis of FRET signals for subcellular cAMP quantification,” Methods in Molecular Biology (Clifton, N.J.) 1294, 59-70 (2015).

[15]. Rich TC, Britain AL, Stedman T, and Leavesley SJ, "Hyperspectral imaging of FRET-based cGMP probes," Methods in Molecular Biology (Clifton, N.J.) 1020, 73-88 (2013).

[16]. Klarenbeek J, Goedhart J, Batenburg A van Groenewald D, and Jalink K, "Fourth-Generation Epac-Based FRET Sensors for cAMP Feature Exceptional Brightness, Photostability and Dynamic Range: Characterization of Dedicated Sensors for FLIM, for Ratiometry and with High Affinity," PLOS ONE 10(4), e0122513 (2015). [PubMed: 25875503] 


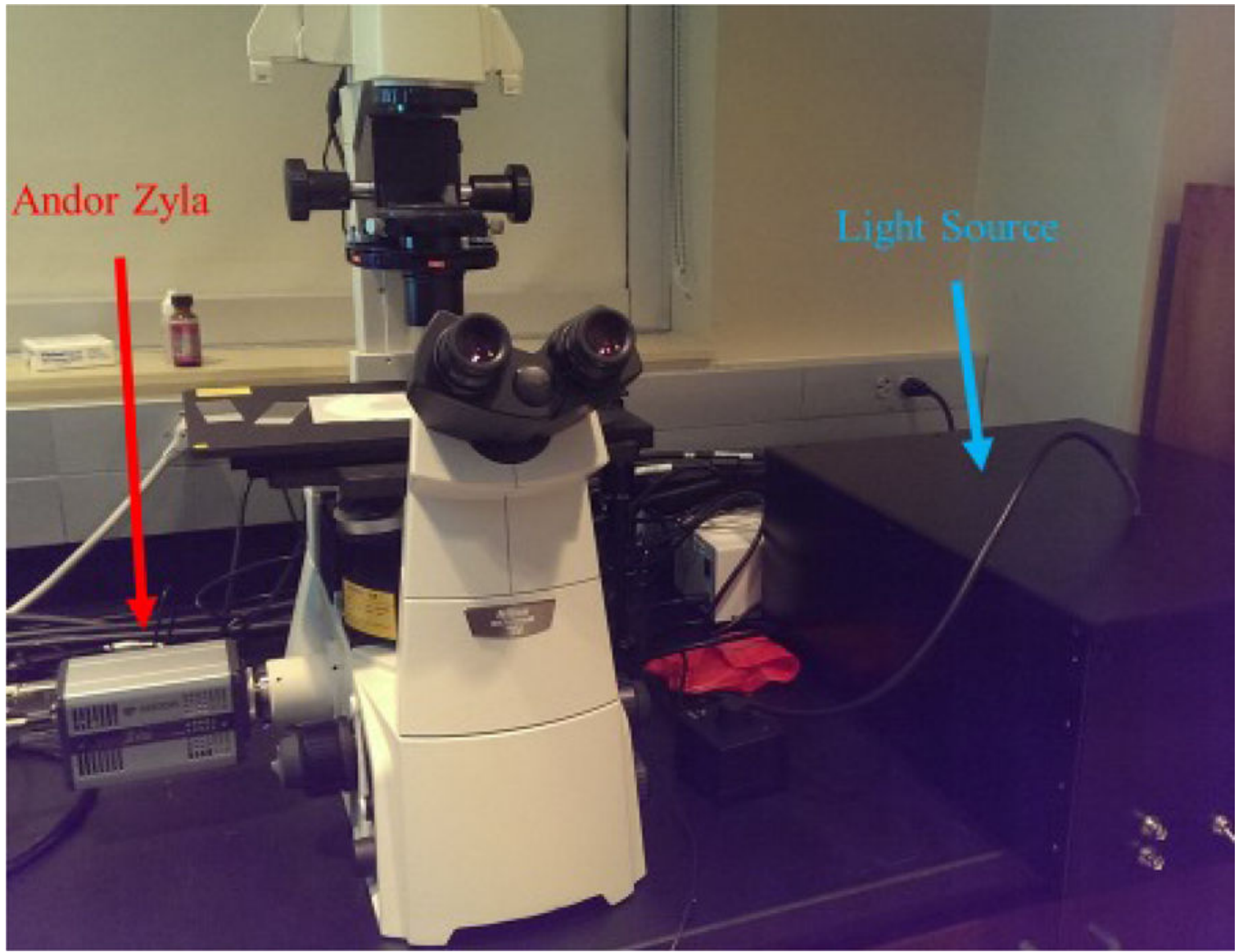

Figure 1 -.

A photograph of the beta prototype system is shown above. The custom high-speed hyperspectral illumination source (at right) produces illumination that is passed through a liquid light guide into a Nikon Eclipse Ti microscope. The illumination reflects off a dichroic filter up through a 60x objective to the stage. An Andor Zyla sCMOS camera is connected to the left port on the microscope to acquire images. 


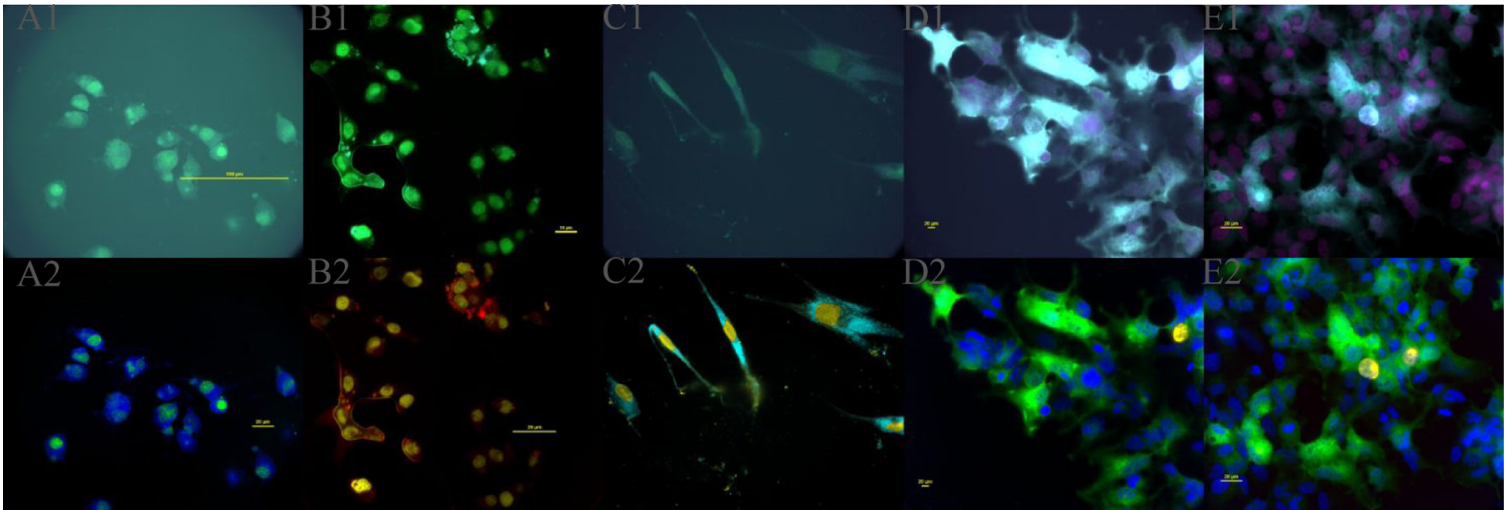

Figure 2 -.

For all panels in this set the top row (1) are raw im ages or treated as spectral (NIS Elements). The panels in the bot tom row (2) are unmixed. Panels: A1 \& A2) MVEC la beled with H188 Turquoise-Venus FRET reporter and DRAQ5. B1 \& B2) MVE C labeled with H 188 Turquoise-Venus FRET reporter and DRAQ5. C1 \& C2) H ASMC labeled with H188 Turq uoise-Venus FR ET reporter and D RAQ5. D1 \& D 2) HEK293 lab eled with H188 Turquoise-Venu s FRET reporter, Nuc Blue, W GA, Calcein Green, and Tubilin Track er. E1 \& E2) HE K293 labeled w ith H188 Turquoise-Venus FRE T reporter, Nuc Blue, WGA, Calcein Green, and Tubilin Tracker. 


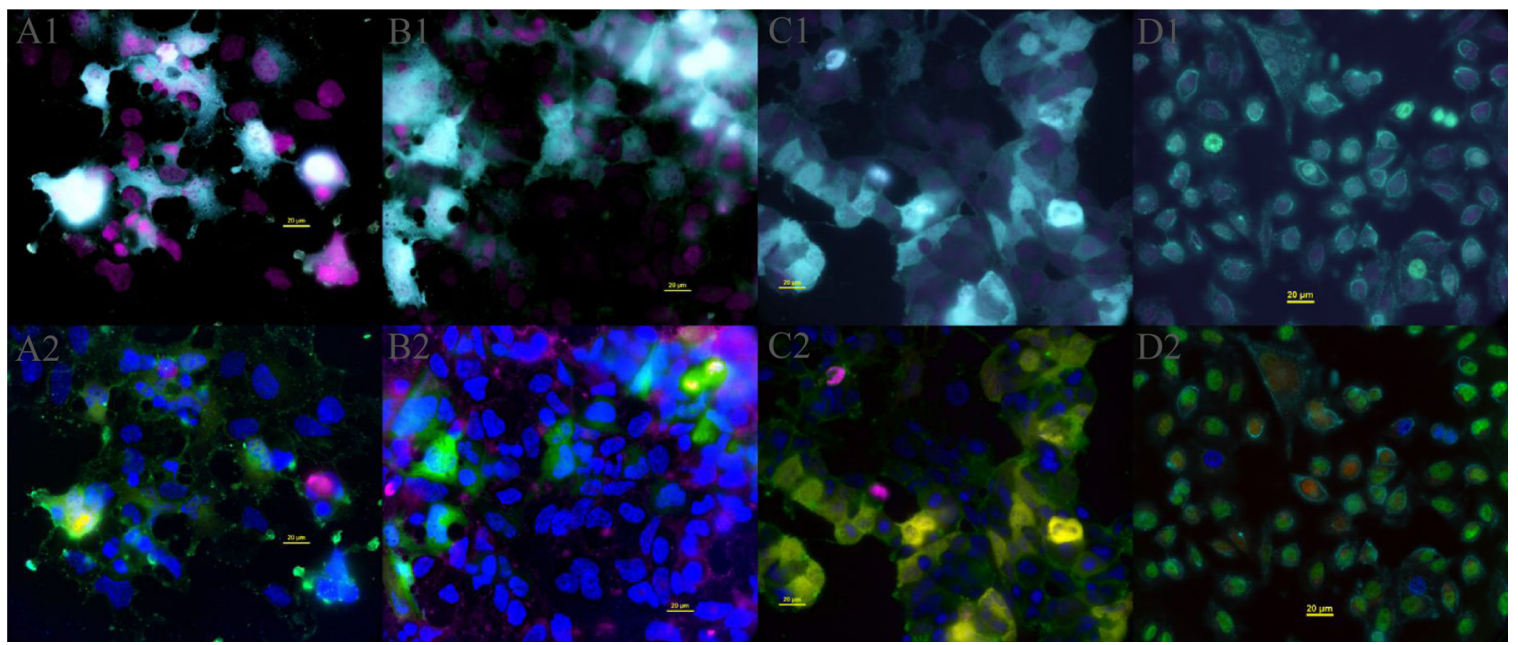

Figure 3 -.

For all panels in this set the top row (1) are treated as spectral (NIS Elements). The panels in the b ottom row (2) are unmixed. Pan els: A1 \& A2) H EK293 labeled with H188 TurquoiseVenus FR ET reporter, Nu c Blue, WGA, and Calcein Green. B1 \& B2) HEK293 1 abeled with H1 88 Turquoise-V enus FRET reporter, Nuc Blue, WGA, and Calcein Green. C1 \& C2) HEK293 labeled with H188 Turquoise-Venus F RET reporter, Nuc Blue, WGA, Calcein Green, and Tubilin Tr acker. D1 \& D2 ) Human Melan oma labeled with H 188 TurquoiseVenus FRET reporter, NucBlu, Calcein Green, and WGA. 